\title{
DETERMINATION OF HEAVY METALS CONCENTRATION IN WINE USING INDUCTIVELY COUPLED PLASMA MASS SPECTROMETRY (ICP-MS)
}

\author{
F.D. Bora1*, A. Ciubucă11, G. Tăbăranu1, V. Enache1, 0. Cioroi1', A. Murăraşu1, \\ G.G. Ciocan'1and S.N. Noapteș ${ }^{1}$
}

1Research and Development Station for Viticulture and Oenology Bujoru, Galaţi, Romania

* Corresponding author: boraflorindumitru@gmail.com

\begin{abstract}
The determination of metals in different type of wines is of high importance for the nutritional, as well as toxic effects of these elements. The main purpose of this research is to determine the concentration of $\mathrm{Cu}, \mathrm{Fe}, \mathrm{Mn}, \mathrm{Cd}, \mathrm{Zn}$ and $\mathrm{Pb}$ in wine. The analyzed samples were wine samples obtained under micro-vinification conditions and from grapevine cultivars for the white wines (Feteasca Alba, Feteasca Regala, Babeasca Gri, Sarba) and for the red wines (Merlot, Feteasca Neagră, Cabernet Sauvignon). The highest concentrations were obtained in the cultivars of grapevine for the red wines $(49.86 \pm 1.32 \mu \mathrm{g} / \mathrm{L} \mathrm{Pb})$ recorded by the Feteasca Neagra cultivar and $(0.23 \pm 0.01 \mu \mathrm{g} / \mathrm{L} \mathrm{Cd})$ for the Merlot cultivar. In the case of $\mathrm{Cu}, \mathrm{Zn}, \mathrm{Fe}$ and $\mathrm{Mn}$, the highest concentrations were recorded by the vines for white wines (532.48 \pm 13.79 $\mu \mathrm{g} / \mathrm{L} \mathrm{Cu}, 1845.23 \pm 32.58 \mu \mathrm{g} / \mathrm{L} \mathrm{Zn}, 1654.98 \pm 26.68 \mu \mathrm{g} / \mathrm{L} \mathrm{Fe}, 221.32 \pm 17.49 \mu \mathrm{g} / \mathrm{L} \mathrm{Mn})$ in comparison to the cultivars of vines for red wines. The conclusions obtained from the analysis of heavy metals in wines through ICP-MS are as follows: grapevine cultivars for red wine recorded the highest concentrations of $\mathrm{Cd}$ and $\mathrm{Pb}$ and the grapevine cultivars for white wines recorded the highest concentrations of $\mathrm{Cu}, \mathrm{Zn}, \mathrm{Fe}$ and Mn.Wine samples do not have a metal ion concentration higher than the maximum concentration allowed by law.The storage temperature does not affect the concentration of heavy metals, dry wine has a higher concentration of heavy metals in its composition.
\end{abstract}

Keywords: heavy metals, wine, ICP-MS, DealuBujorului, Romania

\section{INTRODUCTION}

A subject on the daily agenda for modern oenology is the presence of contaminating metals, especially of heavy metals in the wines (Bora et al., 2018). Thus, nowadays, there is a great interest in identifying the various sources leading to the presence of metals in wine, aiming to reducing the content of these metals by using various treatments permitted by the legislation in force. It is necessary to realistically know the influence of various factors of endogenous nature, such as the grape cultivar, the location of the vineyards, the soil, the climatic conditions of the year (Zinicovscaiaet al., 2017). It is also necessary to know, as much as possible, the influence of various factors of exogenous nature: conditions of harvesting, vinification technology, various enological practices applied, conditions for wines preserving (Bora et al., 2018). The wine samples were obtained under micro- 
vinification conditions and were made of vine cultivars for white wines (Fetească Albă, Fetească Regală, Băbească Gri, Șarbă) and red wines (Merlot, Fetească Neagră, Cabernet Sauvignon).

\section{MATERIALS AND METHODS}

Two types of wine (dry and semi-dry wine) were analyzed, as well as the influence of storage temperature $\left(25^{\circ} \mathrm{C}\right.$ and $\left.5{ }^{\circ} \mathrm{C}\right)$ on the content of metal ions. The wine samples were obtained under micro-vinification conditions and consisted of grapevine cultivars for white wines (Feteasca Alba, FeteascaRegala, Băbeascagri, Șarba) and for red wines (Merlot, FeteascaNeagra, Cabernet Sauvignon), obtained under the ecoclimatic conditions of the years 2015 and 2016. The determination of $\mathrm{Cu}, \mathrm{Fe}, \mathrm{Mn}, \mathrm{Cd}, \mathrm{Zn}$ and $\mathrm{Pb}$ was performed using inductively coupled plasma mass spectrometry (ICP-MS). The ICP-MS calibration operation was performed using the standard XXI CertiPUR multi-element solution (Merck), and the calibration operation was performed on a daily basis. The accuracy of the used method was evaluated through repeated analysis of solutions with known concentration, the obtained results ranged between $0.8-13.1 \%$ depending on each analyzed element. The method's recovery rate for each item ranged from $84.6-99.8 \%$. The wine samples preparation was made by measuring a quantity of $0.2 \mathrm{~mL}$ of the sample over which $8 \mathrm{~mL}$ of reagent $(7 \mathrm{~mL}$ $69 \% \mathrm{HNO}_{3}$ and $1 \mathrm{~mL} \mathrm{H}_{2} \mathrm{O}_{2} \%$ ) is added and placed directly into the digestion vessel of the Milestone START D Microwave Digestion System, waited for 15 minutes, then digested according to the Milestone Digestion System parameters shown in Table 2. After digestion, the samples are transferred to a $50 \mathrm{~mL}$ volumetric flask, with $1 \% \mathrm{HNO}_{3}$ for preservation.

The detection limit (LoD) and the limit of quantification (LoQ) were calculated using the following formula $\mathrm{LoD}=3 \mathrm{SD} / \mathrm{s}$ and $\mathrm{LoQ}=10 \mathrm{SD} / \mathrm{s}(\mathrm{SD}=$ standard deviation, $\mathrm{s}=$ calibration curve). Table 1 displays the calibration conditions for the ICP-MS.

Table 1. Calibration conditions (LoD, LoQ, BEC) of ICP-MS device

\begin{tabular}{|c|c|c|c|c|}
\hline Element & Correlation coefficient & LoD $(\mu \mathrm{g} / \mathrm{L})$ & LoQ $(\mu \mathrm{g} / \mathrm{L})$ & BEC $(\mu \mathrm{g} / \mathrm{L})$ \\
\hline $\mathrm{Cu}$ & 0.9999 & 0.0402 & 0.1339 & 0.237 \\
\hline $\mathrm{Fe}$ & 0.9999 & 5.2102 & 17.3500 & 71.399 \\
\hline $\mathrm{Mn}$ & 0.9999 & 0.0102 & 0.0340 & 0.085 \\
\hline $\mathrm{Cd}$ & 0.9999 & 0.0202 & 0.0673 & 0.027 \\
\hline $\mathrm{Zn}$ & 0.9999 & 0.3780 & 1.2587 & 5.401 \\
\hline $\mathrm{Pb}$ & 0.9999 & 0.0003 & 0.0010 & 0.002 \\
\hline
\end{tabular}

$\mathrm{BEC}=$ background correction .

Table 2. Digestion conditions of the Milestone START D Microwave Digestion System device

\begin{tabular}{|c|c|c|c|c|c|}
\hline \multicolumn{5}{|c|}{ Wine } \\
\hline Step & Time & Airing & Temperature $\left({ }^{\circ} \mathrm{C}\right)$ & Pressure $(\mathrm{Pa})$ & Power $(\mathrm{W})$ \\
\hline I & $00: 10: 00$ & - & 200 & - & 1000 \\
\hline II & $00: 15: 00$ & - & 200 & - & 1000 \\
\hline III & $00: 60: 00$ & + & 35 & - & 0 \\
\hline
\end{tabular}

Effective metal determination was performed using an inductively coupled plasma mass spectrometer (ICP-MS) iCAP Q from Thermo Scientific, a quadrupole model for the most efficient separation of the elements. The device was daily optimized, the carrier gas was Argon 5.0 with 99.99\% purity (Messer). The statistical analysis of the results was carried out using the SPSS (Statistical Package for Social Sciences) version 24. 


\section{RESULTS AND DISCUSSIONS}

As can be seen, the concentration of $\mathrm{Cu}$ varies within wide limits, the highest concentration was recorded in Fetească Albă (532.48 $\pm 13.79 \mu \mathrm{g} / \mathrm{l})$ and Fetească Regală $(511.21 \pm 1.70 \mu \mathrm{g} / \mathrm{L})$, both from the production year of 2015. Of the grapevine cultivars for the red wines, the Cabernet Sauvignon cultivar from 2015 is distinguished, with the highest concentration of $\mathrm{Cu}(496.96 \pm 4.95 \mu \mathrm{g} / \mathrm{L})$. The white wines record $383.08 \mu \mathrm{g} / \mathrm{L}$ (mean value) while the red wines record $380.02 \mu \mathrm{g} / \mathrm{L}$ (mean value), statistically there is no difference between the two variants. The $\mathrm{Cu}$ distribution was significantly influenced by the crop year and by the interaction of the cultivar factor with the crop year (Table 3).As in the case of $\mathrm{Cu}$ and Fe, the highest values are recorded for the vine cultivars for the white wines (Feteasca Alba $1556.80 \pm 29.49 \mu \mathrm{g} / \mathrm{L}$ and Băbească gri $1654.98 \pm 36.17 \mu \mathrm{g} / \mathrm{L}$, culture year 2015), while the grapevine cultivars for the red wines recorded the lowest concentrations (Cabernet Sauvignon $996.61 \pm 8.11 \mu \mathrm{g} / \mathrm{L}$ from 2016). The distribution of Fe was significantly influenced by the crop year and also by the interaction between the cultivar factor and crop year (Table 3). The Feteasca Regală cultivars $(225.32 \pm 9.15 \mu \mathrm{g} / \mathrm{L}$ ) and Merlot (212.49 $\pm 2.12 \mu \mathrm{g} / \mathrm{L})$, from the crop year of 2015 and respectively, 2016 recorded the highest concentrations of $\mathrm{Mn}$ in comparison with the cultivars of BăbeascaGri from 2016 $(119.19 \pm 6.89 \mu \mathrm{g} / \mathrm{L})$ and Cabernet Sauvignon (115.65 $\pm 5.06 \mu \mathrm{g} / \mathrm{L})$ from 2015 , which recorded the lowest concentrations of $\mathrm{Mn}$. The white wines recorded $180.73 \mu \mathrm{g} / \mathrm{L}$ (mean value) while red wines recorded $162.65 \mu \mathrm{g} / \mathrm{L}$ (mean value); from the statistical point of view, there is a significant difference between the two variants, therefore, can be said that the white wine has a higher concentration of Mn than the red one (Bora al., 2018). The distribution of Mn was significantly influenced by the crop year, by the interaction of the cultivar factor and the crop year, whereas the interaction between the cultivar and the storage temperature, of year and storage temperature had a significant influence regarding this parameter (Table 3).

In the analyzed samples of wine, the concentration of $\mathrm{Cd}$ is very low. Higher concentrations were recorded in the Feteasca Albă cultivars $(0.09 \pm 0.01 \mu \mathrm{g} / \mathrm{L})$ and Merlot $(0.09 \pm 0.01 \mu \mathrm{g} / \mathrm{L})$, followed by the Feteasca Regală and Șarba cultivars. In this case, as can be seen, the Cd concentration was extremely low, in most of the analyzed cultivars this concentration was below the limit of detection (Table 3). The $\mathrm{Cd}$ distribution was significantly influenced by the interaction between the cultivar factor and crop year.

Higher concentrations of $\mathrm{Zn}$ were identified in the Sarba cultivar in both years of culture $(1845.23 \pm 9.90 \mu \mathrm{g} / \mathrm{L}$ and $1758.54 \pm 18.86 \mu \mathrm{g} / \mathrm{L})$. The distribution of $\mathrm{Zn}$ was significantly influenced by the crop year, by the interaction of the cultivar factor with the crop year, while the interaction between cultivar and storage temperature, year and storage temperature both had a significant influence on this parameter (Table 3). The grapevine cultivars for red wines recorded the highest values, especially the FeteascaNeagracultivar $(49.86 \pm 1.69 \mu \mathrm{g} / \mathrm{L})$ followed by the Cabernet Sauvignon cultivar $(40.23 \pm 3.00 \mu \mathrm{g} / \mathrm{l})$. White wines recorded $6.87 \mu \mathrm{g} / \mathrm{L}$ (mean value), while red wines recorded $36.91 \mu \mathrm{g} / \mathrm{L}($ mean value), there is a statistically significant difference between the two variants, thereby, the white wine can be said to have a higher concentration of $\mathrm{Pb}$ than the red one. For Băbeasca gri and Feteasca Regală, the concentration of $\mathrm{Pb}$ is below the detection limit. The $\mathrm{Pb}$ distribution was significantly influenced by the crop year, by the interaction between the cultivar factor and crop year, whereas the interaction between the cultivar and the storage temperature, year and storage temperature had a significant influence on this parameter (Table 3, 4).

As can be seen in Figure 1, the concentration of the determined heavy metals has been reported to the international law so that it can be properly ascertained if the wine samples 
can be consumed without having a negative impact on the consumer. In the case of $\mathrm{Cu}, \mathrm{Fe}$, $\mathrm{Mn}, \mathrm{Cd}, \mathrm{Zn}$ and $\mathrm{Pb}$ concentration there were no wine samples found to exceed the maximum permitted by law. Moreover, for both $\mathrm{Cd}$ and $\mathrm{Pb}$, the obtained results are significantly lower than the values provided by the law, and for the $\mathrm{Cu}, \mathrm{Fe}, \mathrm{Mn}$ and $\mathrm{Zn}$ the values were close to these limits.

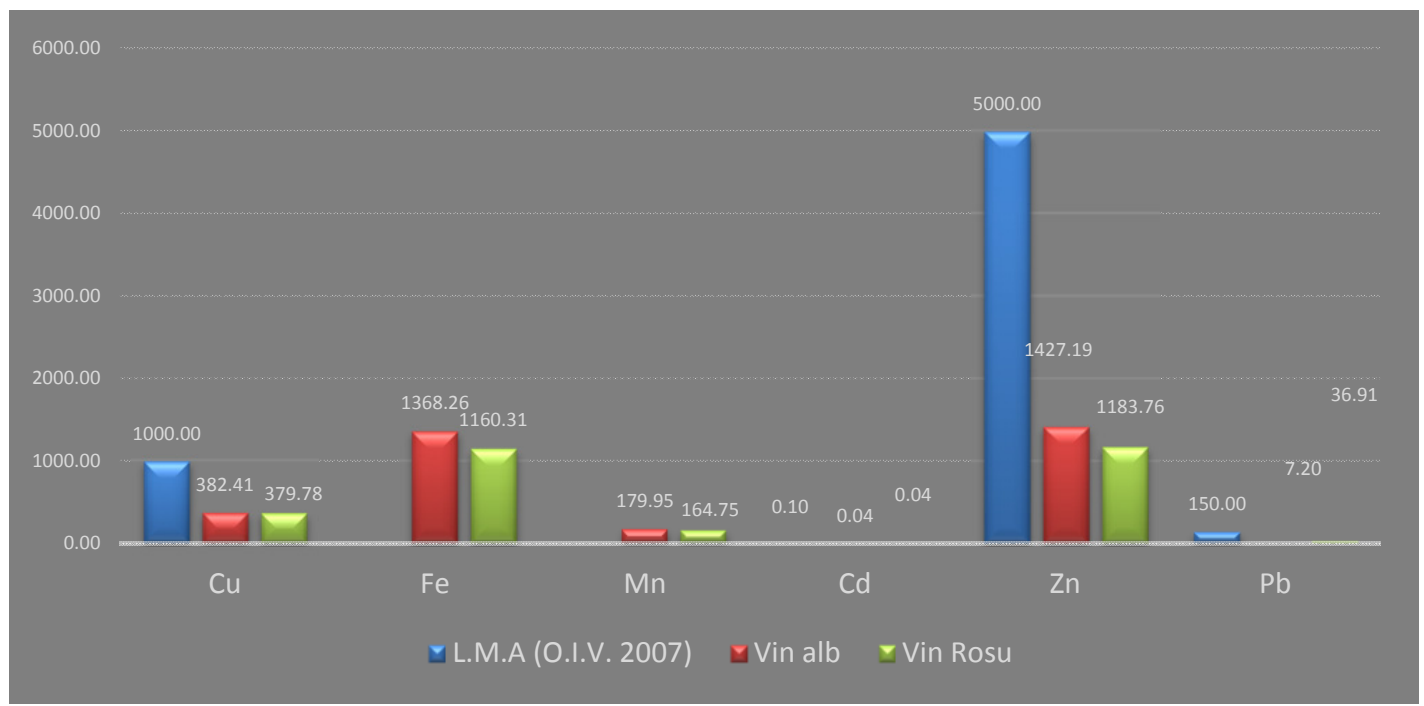

Figure 1. Reporting the obtained results to the Maximum Allowed Limits provided in O.I.V. 2007 $(\mu \mathrm{g} / \mathrm{l})$

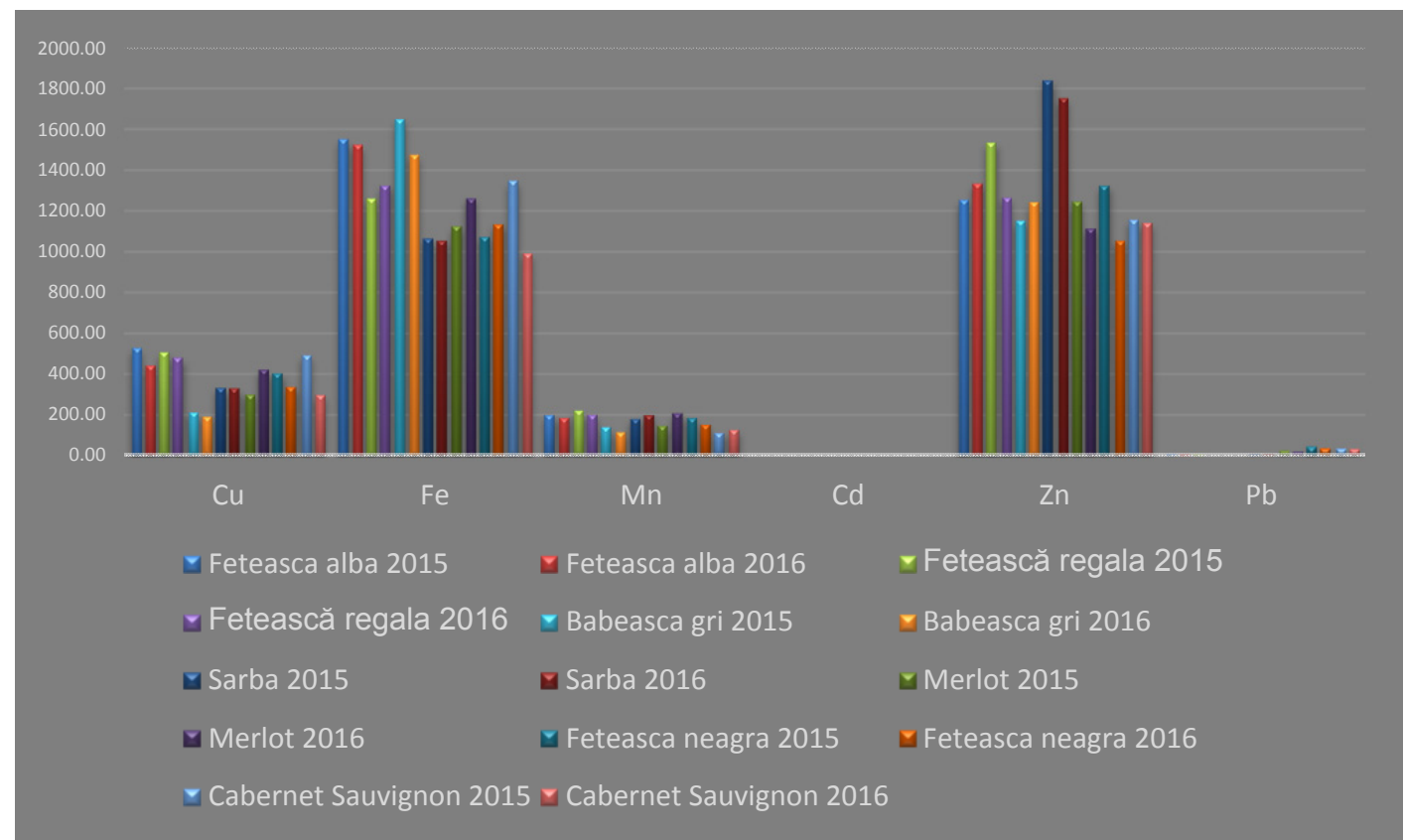

Figure 2. Influence of storage temperature $\left(5^{\circ} \mathrm{C}\right)$ on heavy metals concentration in wine $(\mu \mathrm{g} / \mathrm{l})$ 


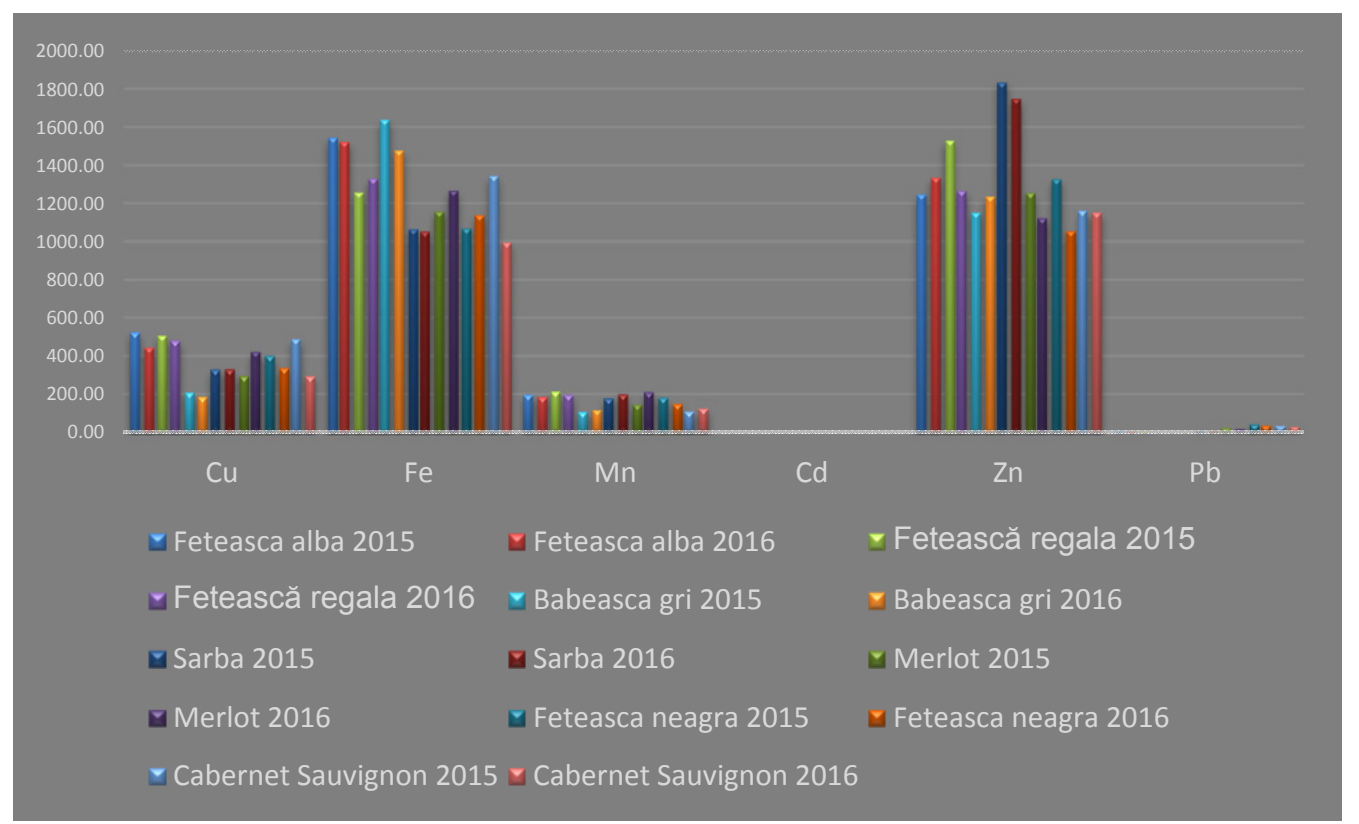

Figure 3. Influence of storage temperature $\left(25^{\circ} \mathrm{C}\right)$ on the concentration of heavy metals in wine $(\mu \mathrm{g} / \mathrm{l})$

The storage temperature for the samples of wine had no influence on heavy metals. In both cases, their concentration was the same, with few exceptions that could be attributed to storage vessels and not to storage temperature (Figure 2 and 3 ).

Table 3. Concentration of heavy metals in wine.

\begin{tabular}{|c|c|c|c|c|c|}
\hline Cultivar & Year & $\begin{array}{c}\text { Storage } \\
\text { Temp }\end{array}$ & $\begin{array}{c}\mathrm{Cu} \\
\mu \mathrm{g} / \mathrm{l}\end{array}$ & $\begin{array}{c}\mathrm{Fe} \\
\mu \mathrm{g} / \mathrm{l}\end{array}$ & $\begin{array}{l}\text { Mn } \\
\mu \mathrm{g} / \mathrm{l}\end{array}$ \\
\hline \multicolumn{3}{|c|}{ Maximum permitted limit } & $1 \mathrm{mg} / \mathrm{l}$ & - & - \\
\hline \multirow{2}{*}{ Feteasca Alba } & $\begin{array}{l}\text { 2015Semi- } \\
\text { dry }\end{array}$ & \multirow{11}{*}{$5^{\circ} \mathrm{C}$} & $\begin{array}{c}532.48 \pm 13.79 \mathrm{a} \\
\alpha\end{array}$ & $\begin{array}{c}1556.80 \pm 29.49 \mathrm{~b} \\
\beta\end{array}$ & $\begin{array}{c}203.53 \pm 7.42 \mathrm{c} \\
\beta\end{array}$ \\
\hline & 2016Dry & & $\begin{array}{c}446.39 \pm 13.48 \mathrm{~d} \\
\delta\end{array}$ & $1529.79 \pm 7.77 \mathrm{~b} \beta$ & $\begin{array}{c}188.91 \pm 1.18 \mathrm{~d} \\
\gamma\end{array}$ \\
\hline \multirow{2}{*}{$\begin{array}{c}\text { Feteasca } \\
\text { Regala }\end{array}$} & $\begin{array}{l}\text { 2015Semi- } \\
\text { dry }\end{array}$ & & $511.21 \pm 1.70 \mathrm{~b} \beta$ & $\begin{array}{c}1265.78 \pm 29.75 \mathrm{e} \\
\varepsilon\end{array}$ & $\begin{array}{c}225.32 \pm 9.15 \mathrm{a} \\
\alpha\end{array}$ \\
\hline & 2016Dry & & $485.32 \pm 7.15 \mathrm{c} \gamma$ & $1329.49 \pm 4.67 \mathrm{~d} \delta$ & $\begin{array}{c}204.62 \pm 5.67 \mathrm{c} \\
\beta\end{array}$ \\
\hline \multirow{2}{*}{ Babeasca gri } & $\begin{array}{l}\text { 2015Semi- } \\
\text { dry }\end{array}$ & & $216.31 \pm 5.81 \mathrm{i} \iota$ & $\begin{array}{c}1654.98 \pm 36.17 \mathrm{a} \\
\alpha\end{array}$ & $\begin{array}{c}144.24 \pm 10.55 \\
\varepsilon\end{array}$ \\
\hline & $\begin{array}{l}\text { 2016Semi- } \\
\text { dry }\end{array}$ & & $195.39 \pm 5.48 \mathrm{j} \kappa$ & $1480.72 \pm 8.48 \mathrm{c} \gamma$ & $\begin{array}{c}119.19 \pm 6.89 \mathrm{~h} \\
\eta\end{array}$ \\
\hline \multirow{2}{*}{ Șarba } & $\begin{array}{l}\text { 2015Semi- } \\
\text { dry }\end{array}$ & & $336.74 \pm 7.65 \mathrm{~g} \eta$ & $\begin{array}{c}1069.59 \pm 33.24 \mathrm{~g} \\
\eta\end{array}$ & $\begin{array}{c}184.42 \pm 8.89 \mathrm{~d} \\
\gamma\end{array}$ \\
\hline & $\begin{array}{l}\text { 2016Semi- } \\
\text { dry }\end{array}$ & & $335.43 \pm 5.59 \mathrm{~g} \eta$ & $1058.91 \pm 8.88 \mathrm{~g} \eta$ & $\begin{array}{c}203.39 \pm 4.33 \mathrm{c} \\
\beta\end{array}$ \\
\hline \multirow{2}{*}{ Merlot } & 2015Dry & & $303.92 \pm 6.52 \mathrm{~h} \theta$ & $1129.32 \pm 9.18 \mathrm{f} \zeta$ & $\begin{array}{c}150.74 \pm 2.12 \\
\text { ef } \delta \varepsilon\end{array}$ \\
\hline & 2016Dry & & $426.78 \pm 5.11$ e $\varepsilon$ & $\begin{array}{c}1266.13 \pm 47.65 \mathrm{e} \\
\varepsilon\end{array}$ & $\begin{array}{c}212.49 \pm 2.12 \\
\mathrm{bc} \beta\end{array}$ \\
\hline $\begin{array}{c}\text { Feteasca } \\
\text { Neagra }\end{array}$ & 2015Dry & & $408.16 \pm 2.60 \mathrm{f} \zeta$ & $\begin{array}{c}1077.50 \pm 36.73 \mathrm{~g} \\
\eta\end{array}$ & $\begin{array}{c}189.10 \pm 5.00 \mathrm{~d} \\
\gamma\end{array}$ \\
\hline
\end{tabular}




\begin{tabular}{|c|c|c|c|c|c|}
\hline Cultivar & Year & $\begin{array}{c}\text { Storage } \\
\text { Temp }\end{array}$ & $\begin{array}{c}\mathrm{Cu} \\
\mu \mathrm{g} / \mathrm{l}\end{array}$ & $\begin{array}{c}\mathrm{Fe} \\
\mu \mathrm{g} / \mathrm{l}\end{array}$ & $\begin{array}{l}\text { Mn } \\
\mu \mathrm{g} / \mathrm{l}\end{array}$ \\
\hline \multicolumn{3}{|c|}{ Maximum permitted limit } & $1 \mathrm{mg} / \mathrm{l}$ & - & - \\
\hline & $\begin{array}{l}\text { 2016Semi- } \\
\text { dry }\end{array}$ & & $\begin{array}{c}341.30 \pm 17.29 \mathrm{~g} \\
\eta\end{array}$ & $1138.90 \pm 8.13 \mathrm{f} \zeta$ & $\begin{array}{c}155.76 \pm 8.50 \mathrm{e} \\
\delta\end{array}$ \\
\hline \multirow{2}{*}{$\begin{array}{l}\text { Cabernet } \\
\text { Sauvignon }\end{array}$} & $\begin{array}{l}\text { 2015Semi- } \\
\text { dry }\end{array}$ & & $496.96 \pm 4.95 \mathrm{c} \gamma$ & $1353.40 \pm 5.51 \mathrm{~d} \delta$ & $\begin{array}{c}115.65 \pm 5.06 \mathrm{~h} \\
\eta\end{array}$ \\
\hline & $\begin{array}{l}\text { 2016Semi- } \\
\text { dry }\end{array}$ & & $301.58 \pm 3.37 \mathrm{~h} \theta$ & $996.61 \pm 8.11$ h $\theta$ & $\begin{array}{c}130.04 \pm 2.20 \mathrm{~g} \\
\zeta\end{array}$ \\
\hline \multirow{2}{*}{ Feteasca Alba } & $\begin{array}{l}\text { 2015Semi- } \\
\text { dry }\end{array}$ & \multirow{14}{*}{$25^{\circ} \mathrm{C}$} & $531.91 \pm 2.74$ a $\alpha$ & $1552.07 \pm 5.62 \mathrm{~b} \beta$ & $\begin{array}{c}204.35 \pm 4.99 \mathrm{c} \\
\beta\end{array}$ \\
\hline & $\begin{array}{l}\text { 2016Semi- } \\
\text { dry }\end{array}$ & & $450.96 \pm 6.38 \mathrm{~d} \delta$ & $1530.15 \pm 5.56 \mathrm{~b} \beta$ & $\begin{array}{c}193.25 \pm 9.61 \mathrm{~d} \\
\gamma\end{array}$ \\
\hline \multirow{2}{*}{$\begin{array}{l}\text { Feteasca } \\
\text { Regala }\end{array}$} & $\begin{array}{l}\text { 2015Semi- } \\
\text { dry }\end{array}$ & & $513.93 \pm 4.04 \mathrm{~b} \beta$ & $\begin{array}{c}1263.94 \pm 14.13 \mathrm{e} \\
\varepsilon\end{array}$ & $\begin{array}{c}221.66 \pm 1.45 \\
\mathrm{ab} \alpha\end{array}$ \\
\hline & $\begin{array}{l}\text { 2016Semi- } \\
\text { dry }\end{array}$ & & $488.62 \pm 7.91 \mathrm{c} \gamma$ & $1336.77 \pm 6.80 \mathrm{~d} \delta$ & $\begin{array}{c}203.43 \pm 2.17 \mathrm{c} \\
\beta \\
\end{array}$ \\
\hline \multirow{2}{*}{$\begin{array}{c}\text { Babeasca } \\
\text { gri }\end{array}$} & $\begin{array}{l}\text { 2015Semi- } \\
\text { dry }\end{array}$ & & $216.06 \pm 4.24 \mathrm{i} \iota$ & $\begin{array}{c}1645.70 \pm 19.34 \mathrm{a} \\
\alpha\end{array}$ & $\begin{array}{c}114.24 \pm 5.05 \mathrm{~h} \\
\theta\end{array}$ \\
\hline & $\begin{array}{l}\text { 2016Semi- } \\
\text { dry }\end{array}$ & & $193.16 \pm 4.62 \mathrm{j} \kappa$ & $1485.46 \pm 7.76 \mathrm{c} \gamma$ & $\begin{array}{c}122.96 \pm 4.57 \mathrm{~h} \\
\zeta\end{array}$ \\
\hline \multirow{2}{*}{ Șarba } & $\begin{array}{l}\text { 2015Semi- } \\
\text { dry }\end{array}$ & & $336.81 \pm 4.83 \mathrm{~g} \eta$ & $1071.95 \pm 2.19 \mathrm{~g} \eta$ & $\begin{array}{c}185.66 \pm 1.99 \mathrm{~d} \\
\gamma\end{array}$ \\
\hline & $\begin{array}{l}\text { 2016Semi- } \\
\text { dry }\end{array}$ & & $\begin{array}{c}338.3 .05 \pm 3.05 \mathrm{~g} \\
\eta\end{array}$ & $\begin{array}{c}1059.85 \pm 15.06 \mathrm{~g} \\
\eta\end{array}$ & $\begin{array}{c}206.50 \pm 3.75 \mathrm{c} \\
\beta \\
\end{array}$ \\
\hline \multirow{2}{*}{ Merlot } & 2015Dry & & $302.31 \pm 2.76 \mathrm{~h} \theta$ & $1164.16 \pm 65.30 \mathrm{f} \zeta$ & $\begin{array}{c}151.96 \pm 4.56 \\
\text { ef } \delta\end{array}$ \\
\hline & $\begin{array}{l}\text { 2016Semi- } \\
\text { dry }\end{array}$ & & $429.44 \pm 3.70$ e $\varepsilon$ & $1274.31 \pm 7.25$ e $\varepsilon$ & $\begin{array}{c}218.57 \pm 3.42 \mathrm{a} \\
\alpha \\
\end{array}$ \\
\hline \multirow{2}{*}{$\begin{array}{l}\text { Feteasca } \\
\text { Neagra }\end{array}$} & 2015Dry & & $409.16 \pm 1.54 \mathrm{f} \zeta$ & $1075.40 \pm 4.29 \mathrm{~g} \eta$ & $\begin{array}{c}189.10 \pm 4.03 \mathrm{~d} \\
\gamma\end{array}$ \\
\hline & 2016Dry & & $344.33 \pm 6.22 \mathrm{~g} \eta$ & $1145.47 \pm 5.82 \mathrm{f} \zeta$ & $\begin{array}{c}156.50 \pm 0.61 \mathrm{e} \\
\delta\end{array}$ \\
\hline \multirow{2}{*}{$\begin{array}{l}\text { Cabernet } \\
\text { Sauvignon }\end{array}$} & 2015Dry & & $495.20 \pm 5.69 \mathrm{c} \gamma$ & $1350.96 \pm 8.64 \mathrm{~d} \delta$ & $\begin{array}{c}115.70 \pm 4.58 \mathrm{~h} \\
\eta \theta\end{array}$ \\
\hline & $\begin{array}{l}\text { 2016Semi- } \\
\text { dry }\end{array}$ & & $301.14 \pm 1.46 \mathrm{~h} \theta$ & $1002.02 \pm 3.20 \mathrm{~g} \theta$ & $\begin{array}{c}131.48 \pm 6.07 \mathrm{~g} \\
\varepsilon\end{array}$ \\
\hline \multicolumn{3}{|c|}{ Geana et al., 2013} & 500.57 & & 223.50 \\
\hline \multicolumn{3}{|c|}{ Zinicovscaia et al., 2017} & & 1100 & 1000 \\
\hline \multirow{2}{*}{ Feteasca Alba } & \begin{tabular}{|c|}
2015 Semi \\
-dry \\
\end{tabular} & \multirow{7}{*}{$5^{\circ} \mathrm{C}$} & $0.09 \pm 0.01 \mathrm{a} \alpha$ & $1259.15 \pm 2.70$ ef $\varepsilon$ & $12.50 \pm 1.50 \mathrm{~g} \delta$ \\
\hline & 2016Dry & & $\begin{array}{c}0.07 \pm 0.02 \\
\operatorname{abcd} \alpha \beta\end{array}$ & $\begin{array}{c}1339.22 \pm 13.14 \mathrm{~d} \\
\delta\end{array}$ & $10.80 \pm 0.68 \mathrm{~g} \delta$ \\
\hline \multirow{2}{*}{$\begin{array}{l}\text { Feteasca } \\
\text { Regala }\end{array}$} & $\begin{array}{l}\text { 2015Semi } \\
\text {-dry }\end{array}$ & & $0.06 \pm 0.04 \mathrm{~d} \beta$ & $1539.93 \pm 6.16 \mathrm{c} \gamma$ & $10.69 \pm 1.44 \mathrm{~g} \delta$ \\
\hline & 2016Dry & & UDL & $\begin{array}{c}1270.49 \pm 21.84 \mathrm{e} \\
\varepsilon\end{array}$ & UDL \\
\hline \multirow{2}{*}{ Babeasca gri } & $\begin{array}{l}\text { 2015Semi } \\
\text {-dry }\end{array}$ & & UDL & $\begin{array}{c}1156.75 \pm 34.90 \mathrm{~g} \\
\zeta\end{array}$ & UDL \\
\hline & $\begin{array}{l}\text { 2016Semi } \\
\text {-dry }\end{array}$ & & UDL & $\begin{array}{c}1248.21 \pm 14.74 \\
\text { ef } \varepsilon\end{array}$ & UDL \\
\hline Șarba & $\begin{array}{l}\text { 2015Semi } \\
\text {-dry }\end{array}$ & & UDL & $1845.23 \pm 9.90$ a $\alpha$ & $11.91 \pm 0.57 \mathrm{~g} \delta$ \\
\hline
\end{tabular}




\begin{tabular}{|c|c|c|c|c|c|}
\hline Cultivar & Year & $\begin{array}{c}\text { Storage } \\
\text { Temp }\end{array}$ & $\begin{array}{c}\mathrm{Cu} \\
\mu \mathrm{g} / \mathrm{l}\end{array}$ & $\begin{array}{c}\mathrm{Fe} \\
\mu \mathrm{g} / \mathrm{l}\end{array}$ & $\begin{array}{l}\text { Mn } \\
\mu \mathrm{g} / \mathrm{l}\end{array}$ \\
\hline \multicolumn{3}{|c|}{ Maximum permitted limit } & $1 \mathrm{mg} / \mathrm{l}$ & - & - \\
\hline & $\begin{array}{l}\text { 2016Semi } \\
\text {-dry }\end{array}$ & & $0.08 \pm 0.02 \mathrm{abc} \alpha$ & $\begin{array}{c}1758.54 \pm 18.86 \mathrm{~b} \\
\beta\end{array}$ & $11.68 \pm 0.64 \mathrm{~g} \delta$ \\
\hline \multirow{2}{*}{ Merlot } & 2015Dry & & $0.09 \pm 0.01 \mathrm{ab} \alpha$ & $\begin{array}{c}1251.24 \pm 40.01 \\
\text { ef } \varepsilon\end{array}$ & $29.08 \pm 6.29$ e $\gamma$ \\
\hline & 2016Dry & & $0.09 \pm 0.02 \mathrm{abc} \alpha$ & $1119.11 \pm 6.44 \mathrm{i} \zeta$ & $24.12 \pm 5.77 \mathrm{f} \gamma$ \\
\hline \multirow{2}{*}{$\begin{array}{l}\text { Feteasca } \\
\text { Neagra }\end{array}$} & 2015Dry & & UDL & $1328.72 \pm 3.27 \mathrm{~d} \delta$ & $\begin{array}{c}49.86 \pm 1.69 \mathrm{a} \\
\alpha\end{array}$ \\
\hline & $\begin{array}{l}\text { 2016Semi } \\
\text {-dry }\end{array}$ & & UDL & $1058.40 \pm 4.10 \mathrm{j} \eta$ & $\begin{array}{c}41.20 \pm 4.34 \\
\text { bc } \beta\end{array}$ \\
\hline \multirow{2}{*}{$\begin{array}{l}\text { Cabernet } \\
\text { Sauvignon }\end{array}$} & $\begin{array}{l}\text { 2015Semi } \\
\text {-dry }\end{array}$ & & UDL & $\begin{array}{c}1161.32 \pm 24.35 \mathrm{~g} \\
\zeta\end{array}$ & $\begin{array}{c}40.23 \pm 8.50 \\
\text { bc } \beta\end{array}$ \\
\hline & $\begin{array}{l}\text { 2016Semi } \\
\text {-dry }\end{array}$ & & UDL & $\begin{array}{c}1145.70 \pm 12.29 \\
\operatorname{gh} \zeta \eta\end{array}$ & $\begin{array}{c}36.98 \pm 3.00 \mathrm{~cd} \\
\beta \\
\end{array}$ \\
\hline \multirow{2}{*}{ Feteasca Alba } & $\begin{array}{l}\text { 2015Semi } \\
\text {-dry }\end{array}$ & \multirow{14}{*}{$25^{\circ} \mathrm{C}$} & $\begin{array}{c}0.07 \pm 0.02 \\
\text { abcd } \alpha \beta\end{array}$ & $\begin{array}{c}1254.18 \pm 6.12 \\
\text { ef } \zeta \eta\end{array}$ & $12.84 \pm 0.93 \mathrm{~g} \zeta$ \\
\hline & $\begin{array}{l}\text { 2016Semi } \\
\text {-dry }\end{array}$ & & $\begin{array}{c}0.08 \pm 0.01 \\
\operatorname{abcd} \alpha \beta\end{array}$ & $1341.86 \pm 6.29 \mathrm{~d} \delta$ & $10.83 \pm 0.24 \mathrm{~g} \zeta$ \\
\hline \multirow{2}{*}{$\begin{array}{l}\text { Feteasca } \\
\text { Regala }\end{array}$} & $\begin{array}{l}\text { 2015Semi } \\
\text {-dry }\end{array}$ & & $0.06 \pm 0.02 \mathrm{bcd} \beta$ & $1536.64 \pm 6.60 \mathrm{c} \gamma$ & $10.85 \pm 0.24 \mathrm{~g} \zeta$ \\
\hline & $\begin{array}{l}\text { 2016Semi } \\
\text {-dry }\end{array}$ & & UDL & $1272.90 \pm 6.43$ e $\varepsilon$ & UDL \\
\hline \multirow{2}{*}{$\begin{array}{l}\text { Babeasca } \\
\text { gri }\end{array}$} & $\begin{array}{l}\text { 2015Semi } \\
\text {-dry }\end{array}$ & & UDL & $1157.96 \pm 6.49 \mathrm{~g} \eta$ & UDL \\
\hline & $\begin{array}{l}\text { 2016Semi } \\
\text {-dry }\end{array}$ & & UDL & $1243.45 \pm 4.04 \mathrm{f} \zeta$ & UDL \\
\hline \multirow{2}{*}{ Șarba } & $\begin{array}{l}\text { 2015Semi } \\
\text {-dry }\end{array}$ & & UDL & $1841.59 \pm 2.39$ a $\alpha$ & $11.59 \pm 0.55 \mathrm{~g} \zeta$ \\
\hline & $\begin{array}{l}\text { 2016Semi } \\
\text {-dry }\end{array}$ & & $\begin{array}{c}0.07 \pm 0.02 \\
\operatorname{abcd} \alpha \beta\end{array}$ & $1756.48 \pm 6.78$ b $\beta$ & $12.15 \pm 0.37 \mathrm{~g} \zeta$ \\
\hline \multirow{2}{*}{ Merlot } & 2015Dry & & $\begin{array}{c}0.07 \pm 0.02 \\
\operatorname{abcd} \alpha \beta\end{array}$ & $\begin{array}{c}1262.77 \pm 3.75 \\
\text { ef } \varepsilon \zeta\end{array}$ & $29.46 \pm 0.90$ e $\delta$ \\
\hline & $\begin{array}{l}\text { 2016Semi } \\
\text {-dry }\end{array}$ & & $0.09 \pm 0.01 \mathrm{a} \alpha$ & $\begin{array}{c}1130.61 \pm 14.62 \mathrm{hi} \\
\theta\end{array}$ & $\begin{array}{c}25.73 \pm 0.51 \\
\text { ef } \varepsilon\end{array}$ \\
\hline \multirow{2}{*}{$\begin{array}{l}\text { Feteasca } \\
\text { Neagra }\end{array}$} & 2015Dry & & UDL & $1334.28 \pm 7.23 \mathrm{~d} \delta$ & $\begin{array}{c}49.10 \pm 2.14 \mathrm{a} \\
\alpha\end{array}$ \\
\hline & 2016Dry & & UDL & $1061.52 \pm 1.92 \mathrm{j} \iota$ & $\begin{array}{c}42.12 \pm 0.91 \mathrm{~b} \\
\beta\end{array}$ \\
\hline \multirow{2}{*}{$\begin{array}{l}\text { Cabernet } \\
\text { Sauvignon }\end{array}$} & 2015Dry & & UDL & $1169.08 \pm 9.36 \mathrm{~g} \eta$ & $\begin{array}{c}40.76 \pm 1.22 \\
\text { bc } \beta\end{array}$ \\
\hline & $\begin{array}{l}\text { 2016Semi } \\
\text {-dry }\end{array}$ & & UDL & $1158.92 \pm 3.33 \mathrm{~g} \eta$ & $\begin{array}{c}35.04 \pm 2.86 \mathrm{~d} \\
\gamma \\
\end{array}$ \\
\hline \multicolumn{3}{|c|}{ Bora et al., 2018} & 0.12 & 1867.19 & 35.50 \\
\hline Zinicov & ala et al., 20 & & & 440 & \\
\hline
\end{tabular}

Average value \pm standard deviation $(n=3)$. Romans' and Greek letters represent the significance of the difference (Duncan test, $p<0.05$ ). The difference between any two values, followed by at least one common letter, is insignificant. M.P.L = maximal permissible limit (O.I.V., 2016). ULD = under of limit of detection. ns = insignificant 


\section{CONCLUSIONS}

The experimental data shows that dry wine has the highest heavy metal content. It is also noted that these wines do not have a metal ion content higher than the maximum permitted by law. The conclusions obtained from the analysis of heavy metals in wine by ICP-MS are as follows: the grapevines cultivars for red wines have recorded the highest concentrations of $\mathrm{Cd}$ and $\mathrm{Pb}$, and the grapevine cultivars for white wines recorded the highest concentrations of $\mathrm{Cu}, \mathrm{Zn}, \mathrm{Fe}$ and $\mathrm{Mn}$; the wine samples do not have a metal ion concentration higher than the maximum permitted by law; the storage temperature does not influence the concentration of heavy metals; dry wine has a higher concentration of heavy metals in its composition.

\section{AUTHOR CONTRIBUTIONS}

F.D.B. and C.A. conceived and designed the experiments; F.D.B. performed the sample collection and processing, the determination of heavy metals. F.D.B. and C.A. contributed to statistical analysis and manuscript revision. T.G., E.V., O.C., M.A., C. G.G. and N.S contributes to data analysis and revised the manuscript. All authors have read and agreed to the published version of the manuscript.

\section{REFERENCES}

1. Geana I., Iordache A., Ionete R., Marinescu A. (2013). Geographical origin identification of Romanian wines by IC-MS elemental analysis. Food Chemistry 138: 1125-1134.

2. Geana I., Marinescu A., Iordache A.M., Sandru C., Ionete R.E., Bala C. (2014). Differentiation of Romanian wines on geographical origin and wine cultivar by elemental composition and phenolic compounds. Food Analytical Methods 7(10): 2064-2074.

3. Zinicovscaia I., Duliu O.G., Culiov O.A., Sturza R., Bilici C., Gundorina S. (2017). Geographical origin identification of Moldavian wines by neutron activation analysis. Food Analytical Methods 10(11): 35233530.

4. Voica C., Deheleanu A., Pamula A. (2009). Method validation for determination of heavy metals in wines and slightly alcoholic beverages by ICP-MS. Journal of Phycics: Conference Series 182: 1-5.

5. Avram V., Voica C., Hosu A., Cimpoiu C., Măruțoiu C. (2014). ICP-MS characterization of some Roumanian white wines by their mineral content. Revue Roumaine de Chimie 59(11-12): 1009-1019.

6. Bora F.D., Donici A., Rusu T., Bunea A., Popescu D., Bunea C.I. (2018). Elemental profile and ${ }^{207} \mathrm{~Pb} /{ }^{206} \mathrm{~Pb}$, ${ }^{208} \mathrm{~Pb} /{ }^{206} \mathrm{~Pb},{ }^{206} \mathrm{~Pb} /{ }^{206} \mathrm{~Pb},{ }^{87} \mathrm{Sr} /{ }^{86} \mathrm{Sr}$ isotope ratio as fingerpints for geographical traceability of Romanian wines. Notuale Botanicae Horti Agrobotanici Cluj-Napoca 46(1): 223-239. 